\title{
Erratum to: Patient-Derived Mammosphere and Xenograft Tumour Initiation Correlates with Progression to Metastasis
}

\author{
Rachel Eyre $^{1}$ - Denis G. Alférez ${ }^{1}$ - Kath Spence ${ }^{1}$ Mohamed Kamal ${ }^{1,2}$. \\ Frances L. Shaw ${ }^{1}$ Bruno M. Simões ${ }^{1} \cdot$ Angélica Santiago-Gómez $^{1}$. \\ Aida Sarmiento-Castro ${ }^{1}$. Maria Bramley ${ }^{3}$. Mohammed Absar ${ }^{3} \cdot$ Zahida Saad $^{4}$. \\ Sumohan Chatterjee $^{4}$ - Cliona Kirwan ${ }^{5}$ - Ashu Gandhi ${ }^{5}$. Anne C. Armstrong ${ }^{6}$. \\ Andrew M. Wardley ${ }^{6}$ - Ciara S. O'Brien ${ }^{6}$ Gillian Farnie ${ }^{7}$ - Sacha J. Howell ${ }^{8,6}$. \\ Robert B. Clarke ${ }^{1}$
}

Published online: 4 November 2016

(C) Springer Science+Business Media New York 2016

\section{Erratum to: J Mammary Gland Biol Neoplasia \\ DOI 10.1007/s10911-016-9361-8}

In the original version of this article, Table 1 contained the following errors: "Grade" was incorrectly labelled as "Grade 1 ", "Nodal Involvement" was incorrectly labelled as "Hormone Receptor Status", the ER positive and ER negative labels were reversed, the Her2 positive was labelled as "Po" instead of "Pos", and "Endocrine therapy" was labelled as" "Endoctrine therapy". In Table 2, "Grade" was incorrectly labelled as "Grade 1", and "Hormone Receptor Status" was incorrectly labelled as "Nodal Involvement". In Table 3, "Pathology" was incorrectly labelled as "Pathlofy". The original article was corrected.

The online version of the original article can be found under doi:10.1007 /s10911-016-9361-8

Robert B. Clarke

robert.clarke@manchester.ac.uk

1 Breast Biology Group, Breast Cancer Now Research Unit, Division of Molecular and Clinical Cancer Sciences, Manchester Cancer, Research Centre, University of Manchester, Wilmslow Road, Manchester M20 4QL, UK

2 Department of Zoology, Faculty of Science, University of Benha, Benha, Egypt

3 Pennine Acute Hospitals NHS Trust, Manchester, UK

4 Salford Royal NHS Foundation Trust, Manchester, UK
5 University Hospitals of South Manchester NHS Foundation Trust, Manchester, UK

6 The Christie NHS Foundation Trust, Wilmslow Road, Manchester M20 4BX, UK

Cancer Stem Cell Research, Division of Molecular and Clinical Cancer Sciences, Manchester Cancer Research Centre, University of Manchester, Wilmslow Road, Manchester M20 4QL, UK

8 Breast Cancer Now Research Unit, Division of Molecular and Clinical Cancer Sciences, Manchester Cancer Research Centre, University of Manchester, Wilmslow Road, Manchester M20 4QL, UK 